\title{
WTF, hier spricht die Polizei!!!
}

juwiss.de/27-2017/

von TOBIAS MAST

Der staatliche Sprachstil hat sich in den sozialen Medien erheblich gewandelt. Statt förmlicher Strenge wird heute gefrotzelt und gejuxt. Doch wann schlägt versuchte Bürgernähe in Anbiederung um? Und steht dieser Bereich (verfassungs-)rechtlicher Regulierung offen, oder sollten hier andere Prämissen, etwa die der Effektivität und Wahrnehmbarkeit, dominieren?

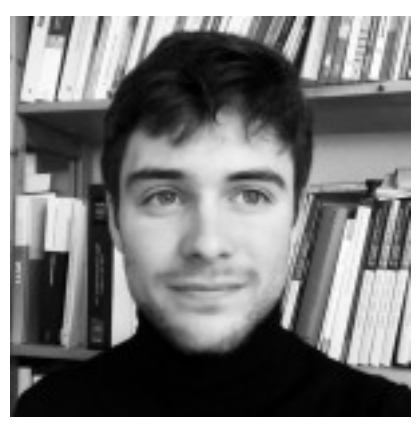

\section{Neuer Stil der Öffentlichkeitsarbeit}

In den letzten Jahren war staatliche Öffentlichkeitsarbeit häufig Gegenstand medialer Berichterstattung, es ging etwa um die Einrichtung des Facebookaccounts der Bundesregierung oder das Vorgehen der Polizeibehörden auf Twitter. Zuletzt sorgte der Twitter-Account der Polizei Mannheim für Aufsehen, nachdem ein Mann in Heidelberg Fußgänger anfuhr. Diese Sachverhalte lenken den Blick auf ein übergeordnetes Phänomen: Staatliche Stellen bedienen sich längst der sozialen Medien für ihre Öffentlichkeitsarbeit. Und wenn man sich die Gefällt mir- (Bundesregierung: über 425000 auf Facebook) und Follower-Zahlen (Polizei Frankfurt: über 170000 auf Twitter) ansieht, verschafft innen das vermutlich eine größere Aufmerksamkeit als jemals zuvor.

Es hat sich aber nicht nur das Medium der Öffentlichkeitsarbeit gewandelt, sondern auch der Stil. Während man bisher eher an förmlich strenge Äußerungen von Behörden gewöhnt war, reiht sich in den sozialen Medien Zwinker-Smily an Großbuchstabenwort. Die Polizei Mannheim rügt einen ausfällig werdenden Nutzer etwa mit den Worten: „Gute Kinderstube vergessen oder nie genossen?". Zur Entkräftung haltloser Spekulationen über Herkunft und Absichten des Heidelberger Autofahrers lässt sie sich gar zu einem „WTF are you talking about?" hinreißen. Wer die einführende Abkürzung nicht kennt, möge sie googeln.

Das Motiv, das hinter diesem neuen Vorgehen steckt, ist eindeutig. Man versucht sich den Rationalitäten der sozialen Medien anzupassen und orientiert sich am Sprachstil der dort erfolgreichen Instanzen. Insofern wird der bereits vor Jahrzehnten eingeschlagene Weg, für staatliche Kommunikation Unterstützung durch private Werbeagenturen einzukaufen, konsequent weitergegangen. Der Grund ist immer noch derselbe: die Angst im Wettstreit um die knappe Ressource Aufmerksamkeit unterzugehen. Und er ist der Sache nach berechtigt und tragfähig. Denn Öffentlichkeitsarbeit dient gesteigerter Transparenz und kann sich so positiv auf Kontrolle, Partizipation und Vertrauen der Gesellschaft auswirken. Sie ist folglich demokratie- und rechtsstaatlich fundiert. 
Dennoch ist Kommunikation im Staat-Bürger-Verhältnis keine von gleich zu gleich. Während sich Private unter dem Schutz der Meinungsfreiheit auch polemisch und unsachlich äußern dürfen, gelten für den Staat andere Prämissen. Regierungen und Behörden kommunizieren um die ihnen obliegenden Aufgaben zu erledigen - nicht aus Jux und Dollerei. Und sie gehen ihren Aufgaben im Rahmen des Rechts nach, auch wenn es auf anderem Wege schneller oder besser ginge.

Aber was sind die verfassungsrechtlichen Vorgaben für Staatskommunikation? Hier betritt man einen nebulösen Bereich. Bisher wurden sie v.a. aus dem Blick der Grundrechtsdogmatik betrachtet. Den Paukenschlag stellten die 2002 zu Warnungen der Bundesregierung vor Frostschutzmittel im Wein (lykol) und einer Sekte (Osho) ergangenen Entscheidungen des BVerfG dar. Seither streitet sich die Rechtswissenschaft: ob der Schutzbereich der Grundrechte denn in jedem Falle vor Information schütze, wann von einem mittelbar-faktischen Grundrechtseingriff durch Information gesprochen werden könne und v.a. ob es für einen solchen einer klassischen Eingriffsermächtigung (=Befugnisnorm) bedürfe.

Nur am Rande werden grundrechtsunabhängige Verfassungsvorgaben erörtert. So stellt das BVerfG in der Glykolentscheidung klar, dass staatliche geäußerte Informationen sachlich und richtig sein müssen. Stimmen aus der Rechtswissenschaft erweitern die inhaltlichen Vorgaben mitunter zu einem bunten Strauß: Sachlich, richtig, vollständig, klar und am besten auch neutral solle sich der Staat äußern. Die Begriffe werden schlagwortartig angeführt, ohne dass ihr Gehalt näher bestimmt würde, und auch über ihre Herleitung herrscht Unklarheit. Angedacht werden etwa Verhältismäßigkeitsprinzip, Willkürverbot und Rechtsstaatsprinzip. Die Ausführungen machen aber deutlich, dass zumindest die Rechtswissenschaft nach wie vor von einer Sonderrolle des Staates, auch bei seiner Kommunikation, ausgeht. Nur ist es schwer, aus derart abstrakten Begriffen konkrete Vorgaben an staatliche Informationstätigkeit abzuleiten. Zumal die technischen Rahmenbedingungen und Eigenrationalitäten des jeweiligen Mediums zu beachten sind. Inhalte funktionieren medienbezogen, Twitter eignet sich nicht für eine ausführliche Hintergrundbeleuchtung der Eurokrise und im gemeindlichen Amtsblatt können interessierte Bürger nicht ihre Meinung unter dem neuesten Bild kundtun.

Die passende normative Vorgabe um den in den sozialen Medien geübten Sprachstil zu disziplinieren, wäre sicherlich das Sachlichkeitsgebot. Dann müsste aber zunächst geklärt werden, ob dieses in einem weiteren Sinne zu verstehen ist und auch Vorgaben an die staatliche Sprache als solche richtet.

Wie sich staatliche Stellen zu artikulieren haben, ist keine statische Entscheidung, sondern steht in Bezug zum gesellschaftlichen Wandel. Eine klinisch-sterile Ausdrucksweise in sozialen Medien zu fordern, wäre absurd. Und doch darf die Sprache nicht beliebig sein, wenn der Staat seine Staatlichkeit auch im Rahmen seiner Kommunikation bewahren will. Wo im Einzelfall die Grenze zwischen bloß unangebrachten und rechtlich unzulässigen Äußerungen gezogen werden soll, ist schwer zu beurteilen. Als verfassungsrechtliche Sprachpolizei aufzutreten, bedeutete eventuell auch Innovations- und Entwicklungspotenziale zu unterdrücken. So wird wohl niemand der Spielverderber sein wollen, wenn die Berliner oder Hamburger Polizei mit lustigen Tweets von sich reden macht. Hier scheinen die sozialen Medien tatsächlich eine Annäherung zwischen Staat und 
Bürgern zu ermöglichen, die das Potenzial in sich trägt, Vertrauen und Akzeptanz zu stärken. Auch gegen den Gebrauch von Smilies wird per se nichts einzuwenden sein. Aber wäre ein Tweet komplett in Großbuchstaben und mit mehreren Ausrufe- bzw. Fragezeichen noch in Ordnung? Und dürfen Nutzer geduzt werden? Vergleichsweise eindeutig sind aus dem Stegreif nur Äußerungen zu beurteilen, die sogar für Private unzulässig wären. Erst dort die Grenze zu ziehen, bedeutete aber u.U. den Vertrauens- und Aufmerksamkeitsvorschuss, der staatlichen Stellen in vielen Bereichen kraft ihrer besonderen Autorität zukommt, zu gefährden. Es erscheint mir zumindest nicht fernliegend, dass die mitunter inhaltlich-thematische Sonderrolle des Staates in den Erwartungen der Rezipienten mit einer gehobenen Seriosität in der Sprachwahl einhergeht. Blind den gesellschaftlichen Selbstdarstellungstrends zu folgen, hieße dann im schlimmsten Falle, die staatlich nicht erzwingbare Aufmerksamkeit als Wirksamkeitsvoraussetzung der immer wichtiger werdenden Handlungsform langfristig aufs Spiel zu setzen.

Deswegen sollte man sich, auch um solche Einzelfragen mit guter Begründung beantworten zu können, zuvor mit Grundsätzlicherem beschäftigen: Hat der Staat unabhängig von Medium und Rechtspositionen Dritter ein absolutes Mindestmaß an Förmlichkeit und Sachlichkeit zu wahren? Darf der Sprachstil anhand des Adressatenkreises variieren? Spielt es eine Rolle, ob die staatliche Stelle zuvor grob angegangen wurde? Und in welchem Verhältnis stehen inhaltliche Vorgaben zu eventuell divergierenden Effektivitätserwartungen?

\section{Fazit}

Nicht jede Frage ist auch eine des Verfassungsrechts. Das Recht darf sich aber auch nicht vorschnell vom oft als unregelbar verschrienen Bereich der Staatskommunikation abwenden. Das Aufkommen der sozialen Medien ging mit der abnehmenden Relevanz medialer Vermittlung für staatliche Informationstätigkeit einher. Dieser Wegfall externer Vorab-Regulierung nach den medienrechtlich gerahmten Rationalitäten des Journalismus muss durch eine stärkere öffentlich-rechtliche Berücksichtigung aufgefangen werden. Dabei darf nicht die Sonderrolle staatlicher Kommunikation im öffentlichen Diskurs vergessen werden. Was für Privatunternehmen eine geeignete Strategie sein mag, muss noch lange nicht für den Staat passen. Im Rennen um die hippste Grafik und den witzigsten Hashtag wird die kleine Fachbehörde niemals mit den Big Playern der PR-Branche wetteifern können. Aber muss sie das? Das BVerfG scheint zumindest für die Regierung von der Bürgererwartung auszugehen, staatliche Informationstätigkeit sei etwas „,besonderes“, das nicht im Wettstreit mit Privaten stehe, sondern als Korrektiv und Ergänzung hierzu zu verstehen sei (BVerfGE 105, 252 (269) und 105, 279 (302)). Soweit ersichtlich, wurde bisher noch nicht empirisch untersucht, inwieweit der gewandelte staatliche Sprachstil tatsächlich der Nutzererwartung entspricht und akzeptanzförderlich ist. Ich wage die Vermutung, dass es auch ohne „WTF“ und Konsorten ginge. 